\title{
Enhanced Phonon Boundary Scattering at High Temperatures in Hierarchically Disordered Nanostructures
}

\author{
DHRITIMAN CHAKRABORTY (1), ${ }^{1,2}$ LAURA DE SOUSA OLIVEIRA, ${ }^{1}$ \\ and NEOPHYTOS NEOPHYTOU ${ }^{1}$ \\ 1.-School of Engineering, University of Warwick, Coventry CV4 7AL, UK. 2.-e-mail: \\ D.Chakraborty@warwick.ac.uk
}

\begin{abstract}
Boundary scattering in hierarchically disordered nanomaterials is an effective way to reduce the thermal conductivity of thermoelectric materials and increase their performance. In this work, we investigate thermal transport in silicon-based nanostructured materials in the presence of nanocrystallinity and nanopores at the range of 300-900 K using a Monte Carlo simulation approach. The thermal conductivity in the presence of nanocrystallinity follows the same reduction trend as in the pristine material. We show, however, that the relative reduction is stronger with temperature in the presence of nanocrystallinity, a consequence of the wavevector-dependent (q-dependent) nature of phonon scattering on the domain boundaries. In particular, as the temperature is raised, the proportion of large wavevector phonons increases. Since these phonons are more susceptible to boundary scattering, we show that this $\boldsymbol{q}$-dependent surface scattering could account for as much as a $\sim 40 \%$ reduction in the thermal conductivity of nanocrystalline Si. The introduction of nanopores with randomized positions magnifies this effect, which suggests that hierarchical nanostructuring is actually more effective at high temperatures than previously thought.
\end{abstract}

Key words: Thermal conductivity, phonon transport, boundary scattering, thermoelectrics, nanotechnology, nanocrystalline silicon, Monte Carlo simulations

\section{INTRODUCTION}

Hierarchical nanostructuring is the most promising approach to date to achieve very low thermal conductivities $(\kappa)$ and high thermoelectric (TE) figures-of-merit $(Z T)$ as hierarchically sized nanofeatures scatter phonons across wavelengths and reduce phonon transport throughout the spectrum. ${ }^{1,2}$ Materials with nanocrystalline boundaries, nanoinclusions, atomic defects, pores, and voids are common practice in new-generation $\mathrm{TE}$ materials. ${ }^{3-7}$ For a PbTe-SrTe system, for example, this method has yielded a lattice thermal conductivity

(Received August 23, 2018; accepted January 12, 2019; published online January 28, 2019)
( $\kappa$ ) of $0.9 \mathrm{Wm}^{-1} \mathrm{~K}^{-1}$ at $915 \mathrm{~K}$ and a $Z T$ of $2.2 .^{2}$ More recent studies have reported an extremely low $\kappa$ of $0.5 \mathrm{~W} \mathrm{~K}^{-1} \mathrm{~m}^{-1}$ and a $Z T$ of 2.5 at $923 \mathrm{~K}$ in a $p$-type $\mathrm{Pb}_{0.98} \mathrm{Na}_{0.02} \mathrm{Te}-\mathrm{SrTe}$ system, ${ }^{8}$ and a similarly low $\kappa=0.55 \mathrm{~W} \mathrm{~K}^{-1} \mathrm{~m}^{-1}$ in a SiGe nanoporous system. ${ }^{7}$ Moreover, our prior works also point out that hierarchical nanostructures can also improve the thermoelectric power factor. ${ }^{9-13}$

From the theoretical point of view, a large number of works by us and others can be found in the literature explaining thermal conductivity trends in nanostructured materials, using a variety of theoretical and simulation methods. ${ }^{14-23}$ In our recent works, for example, we provide a complete roomtemperature analysis of thermal conductivity trends in Si-based nanomaterials in the presence of nanocrystalline boundaries and nanopores. ${ }^{19,22}$ Here, we extend our prior Si-based studies to higher 
temperatures, and focus specifically on answering the following question: are there any quantitative differences in the thermal conductivity trends in hierarchically nanostructured materials between what is observed at room temperature versus what can be observed at higher temperatures? In other words, are nanostructured features more effective or less effective in reducing the thermal conductivity at higher rather than lower temperatures? Our motivation resides on the following: (1) the average mean free path (MFP) for phonon-phonon scattering at room temperature in $\mathrm{Si}$ is indicated in several reports to reside somewhere between $100 \mathrm{~nm}$ and $300 \mathrm{~nm} \cdot{ }^{24-28}$ At elevated temperatures, the MFP is reduced as the Umklapp 3-phonon scattering increases, increasing the importance of phononphonon scattering compared to phonon boundary scattering; and (2), on the other hand, as the temperature is raised, phonons of different frequencies and wavevectors (usually larger wavevectors) participate in transport, which react differently to scattering of the various nanofeatures, and, specifically for this study, of nanocrystalline boundaries (usually increasing scattering probabilities). Therefore, in this work, we focus on understanding how these two seemingly counteracting features affect the thermal conductivity of hierarchically nanostructured materials at higher temperatures. Our results could help in the design of better TE materials with ultra-low thermal conductivities, and the insight we provide could also be generalized for other materials, beyond Si.

\section{APPROACH}

For calculating the thermal conductivity, we solve the phonon Boltzmann transport equation (BTE) using the Monte Carlo method, with all details and validation described in our recent publications, ${ }^{19,20,22}$ and we provide here the method details that are important in this work. The geometries we consider are shown in Fig. 1. For computational efficiency, we consider a $2 \mathrm{D}$ simulation domain of length $L_{\mathrm{x}}=1000 \mathrm{~nm}$ and width $L_{\mathrm{y}}=500 \mathrm{~nm}$, which is adequate for considering all the effects we are investigating. Figure 1a shows a typical nanocrystalline geometry, whereas Fig. 1b shows a typical hierarchically nanostructured geometry, in which nanopores of random position and diameter through a normal distribution are inserted.

In the nanocrystalline geometry cases, the average grain size in the simulation domain is defined as $<d>=L_{\mathrm{x}} /<N_{\mathrm{G}}>$, where $L_{\mathrm{x}}$ is the length of the domain in the transport direction and $\left\langle N_{\mathrm{G}}\right\rangle$ is the average number of grains encountered in that length. Grains in the nanocrystalline case are generated using Voronoi tessellations, where grain boundaries are created by expanding grain areas radially outward from their initial "seeding points" until two areas meet, ${ }^{29}$ given initial input values for the number of "seeding points" and the dimensions of the domain. In these structures, the thermal conductivity is affected in two ways: the scattering of phonons at the grain boundary, and internal three-phonon Umklapp scattering inside the grains. Following the commonly employed boundary scattering picture, the scattering probability at grain boundaries depends on the phonon wave vector, $\boldsymbol{q}$, the roughness of the boundary, $\Delta_{\text {rms }}$, and the angle of incidence between the phonon path and the normal to the grain boundary, $\theta_{\mathrm{GB}}$, as indicated in Fig. 1c. This determines whether an incident phonon will be transmitted to the other side or will be scattered, and is given by the commonly employed relationship ${ }^{29}$ :

$$
t=\exp \left(-4 \boldsymbol{q}^{2} \Delta_{\mathrm{rms}}^{2} \sin ^{2} \theta_{\mathrm{GB}}\right)
$$

If the phonon is scattered, another parameter which depends again on the roughness and the phonon wavevector, the specularity, $p$, determines the angle of phonon scattering. ${ }^{18} p$ takes values from 0 to 1 , with $p=0$ indicating a diffusive, randomized scattering angle where direction of phonon propagation is randomized. Thus, diffusely scattered phonons can also propagate along an angle other than the angle of incidence after scattering. $p=1$ indicates specular reflection where the angle of incidence is the same as the angle of reflection. This is also applied to the boundaries of pores in the nanoporous cases (see Fig. 1b). Specifically, in the case of specular pore boundary scattering, the angle that the phonon will reflect into, $\theta_{\text {ref }}=2 \gamma-\theta_{\text {inc }}$, is defined based on geometrical considerations (the angle of incidence is the same as the angle of reflection) as:

$$
\theta_{\text {ref }}=2 \gamma-\theta_{\text {inc }}
$$

where $\theta_{\text {inc }}$ is the angle of propagation of the phonon relative to the $x$-axis, and $\gamma$ is the angle formed by the line perpendicular to the pore at the point of interaction and the $x$-axis (see Fig. 1d). In the case of the pores, however, we do not explicitly consider the $\boldsymbol{q}$-dependence, but we consider specular or diffusive boundary scattering just by assigning the specularity parameter $p$.

The main investigation we undertake in this work concerns the influence of boundary scattering at high temperatures, and more specifically the role that different wave vector $(\boldsymbol{q})$ and MFP phonons play in grain boundary scattering. Therefore, we find it useful to provide an indication of the participation of larger $\boldsymbol{q}$ phonons in thermal conductivity as the temperature is raised. We can show this for the pristine bulk material based on the lattice thermal conductivity, $\kappa_{l}$, as derived from the Boltzmann transport equation in the single mode relaxation time approximation. The phonon thermal conductivity is given as $\kappa_{l}=\sum_{i, q} \kappa_{l, i}^{\prime}(\boldsymbol{q})$, where ${ }^{30}$ : 

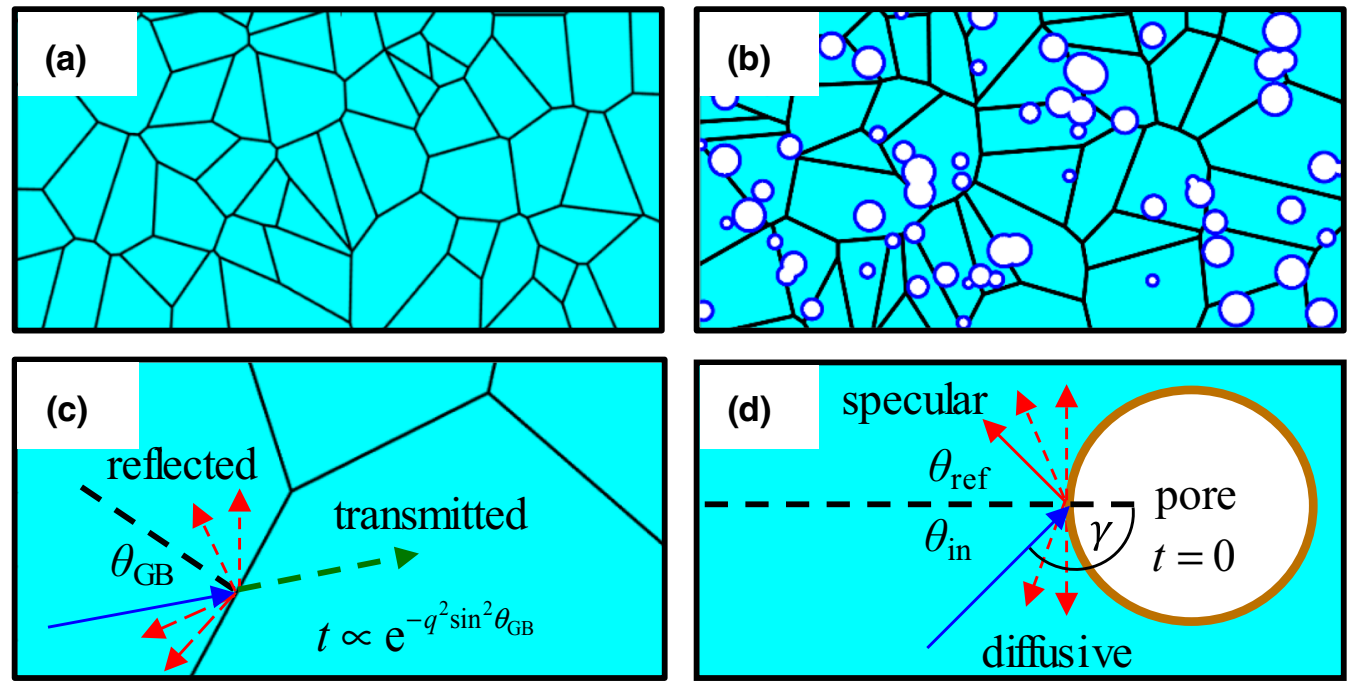

Fig. 1. Examples of the nanostructured geometries considered. (a) Nanocrystalline materials with changing grain dimension $(<d>)$, with $\langle d\rangle=100 \mathrm{~nm}$; black lines represent NC grain edges. (b) Nanocomposite material with given grain dimension $(<d>)$ and porosity $(\phi)$ with $\phi=5 \%$, for a random polydispersed pore arrangement, with pore diameter uniformly distributed between 10 and $50 \mathrm{~nm}$ and random pore position. (c) Schematic for grain scattering indicating the initial angle of the phonon, $\theta_{\mathrm{GB}}$, from the normal (dashed line), grain boundaries (black lines), initial path of the phonon (blue line) and probable paths of the phonon after scattering (red dashed lines and yellow dashed transmitted line); transmission is dependent on grain boundary roughness as well as phonon wave vector $\boldsymbol{q}$, and transmission probability is given by Eq 1 . (d) Schematic for pore scattering indicating the pore boundary, the initial angle of the phonon $\theta_{\text {in }}$, and new angle of propagation after reflection, $\theta_{\text {ref }}$, depending on specularity parameter $p$; probable paths of the phonon after scattering for both diffusive (red dashed lines) and specular (red solid line) are depicted; transmission $(t)$ through the pore is always zero (Color figure online).

$$
\kappa_{l, i}^{\prime}(\boldsymbol{q})=k_{B} \tau_{i}(\boldsymbol{q}) v_{g, i}(\boldsymbol{q})^{2}\left[\frac{\hbar \omega_{i}(\boldsymbol{q})}{k_{B} T}\right]^{2} \frac{e^{\hbar \omega_{i}(\boldsymbol{q}) / k_{B} T}}{\left(e^{\hbar \omega_{i}(\boldsymbol{q}) / k_{B} T}-1\right)^{2}}
$$

In Eq. 3, the index $i$ refers to each mode or band (two transverse and one acoustic), $v_{g, i}(\boldsymbol{q})=$ $\partial \omega_{i}(\boldsymbol{q}) / \partial \boldsymbol{q}$ is the group velocity as a function of the wavevector, and is derived from the expression provided for each band's $\omega, \tau_{i}$ is similarly the relaxation time for each mode, $T$ is the temperature, $k_{B}$ is the Boltzmann constant and $\hbar$ is the reduced Planck's constant. The Bose-Einstein statistics are incorporated in this equation in the so-called phonon window function term ${ }^{30}$ :

$$
W_{p}(\boldsymbol{q})=\frac{3}{\left(\pi^{2} k_{B} T\right)}\left[\frac{\hbar \omega(\boldsymbol{q})}{k_{B} T}\right]^{2} \frac{e^{\hbar \omega(\boldsymbol{q}) / k_{B} T}}{\left(e^{\hbar \omega(\boldsymbol{q}) / k_{B} T}-1\right)^{2}}
$$

which is shown in Fig. 2a, and indicates the part of the phonon spectrum that takes part in transport as the temperature increases. Indeed, the proportion of large $\boldsymbol{q}$ phonons increases (which makes transmission over boundaries on average more difficult; see Eq. 1). On the other hand, since higher energy phonons are excited at higher temperatures, Umklapp scattering increases (decreasing scattering times), which reduces the phonon MFPs. To evaluate the combined effect of higher wavevector phonon participation and additional scattering to the lattice thermal conductivity with temperature, we computed $\kappa_{l, i}^{\prime}$ as in Eq. 3. We adopted the phonon spectrum analytical expression $\omega(\boldsymbol{q})=v_{s} \boldsymbol{q}+\mathbf{c} \boldsymbol{q}^{2}$ with $v_{\mathrm{s}}=9.01 \times 10^{3} \mathrm{~ms}^{-1}$ and $\mathrm{c}=-2 \times 10$ $7 \mathrm{~m}^{2} \mathrm{~s}^{-1}$ for the longitudinal acoustic branch, and $v_{\mathrm{s}}=5.23 \times 10^{3} \mathrm{~ms}^{-1}$ and $\mathrm{c}=-2.26 \times 10^{-7} \mathrm{~m}^{2} \mathrm{~s}^{-1}$ for the transverse acoustic branches, ${ }^{20}$ as used within the Monte Carlo simulator. For simple first-order demonstration for the phonon Umklapp scattering relaxation time, we adopted Holland's model $^{26,30}$ :

$$
\tau_{U}^{-1}=\mathrm{B} \omega_{i}(\boldsymbol{q})^{2} T \exp \left(-\frac{\mathrm{C}}{T}\right)
$$

where $\mathrm{B}=2.8 \times 10^{-19} \mathrm{~s} / \mathrm{K}$ and $\mathrm{C}=140 \mathrm{~K}$. (Note that a more detailed and elaborate scattering rate description is coded in the Monte Carlo solver, as in Refs. 15, 18, 19.

The relative contribution of each $\boldsymbol{q}$-state to the thermal conductivity as the temperature changes, compared to the $300 \mathrm{~K}$ contribution, is shown in Fig. 2b. To produce the plots in Fig. 2b, we first compute $\kappa_{l, i}^{\prime}(\boldsymbol{q})$ at $T=300 \mathrm{~K}, 400 \mathrm{~K}, 500 \mathrm{~K}$, and $600 \mathrm{~K}$ using Eq. 4. Then, we perform the following two steps: (1) we normalize the $\kappa_{l, i}^{\prime}(\boldsymbol{q})$ values with respect to their initial $\boldsymbol{q}=0$ value, $\kappa_{l, i}^{\prime}(0)$, and (2) we normalize those new values with their corresponding values at $300 \mathrm{~K}$ (which we use as our basis). This tells us how much more higher $\boldsymbol{q}$-states contribute to thermal transport at higher temperatures, compared to room temperature. Thus, Fig. $2 \mathrm{~b}$ shows the redistribution of heat flow over the entire spectrum, indicating that high frequency phonon scattering has a greater overall contribution (up to $20 \%$ for $T=600 \mathrm{~K}$ ) to thermal transport at higher temperatures. Note that, although the Window function only captures Bose-Einstein statistics, 

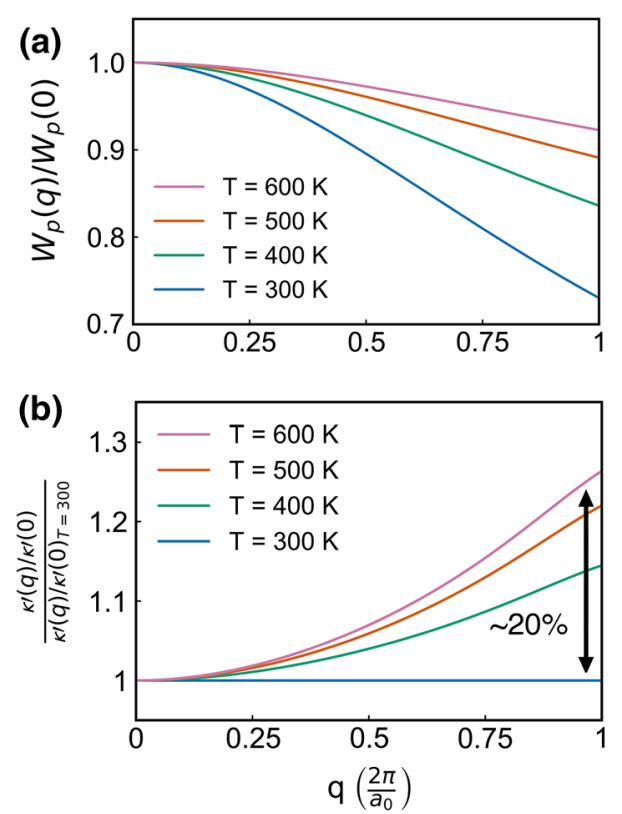

Fig. 2. Change in average phonon attributes with increase in temperature. (a) Phonon window functions versus wavevector (normalized to unity) illustrating the range of phonon wavevectors active at different temperatures; As the temperature increases the window function widens from the blue line at $300 \mathrm{~K}$ to the purple line at $600 \mathrm{~K}$ as the proportion of larger wavevector (shorter wavelength) phonons increases. (b) An indication of the redistribution of heat flow in the phonon spectrum, which is an indication of how much more higher $q$-states contribute to thermal transport at higher temperatures, compared to room temperature. We show normalized $\boldsymbol{q}$-resolved thermal conductivity by its $\kappa_{l, i}^{\prime}(0)$ value, and further normalized by the corresponding $300 \mathrm{~K}$ values. Temperatures $T=400 \mathrm{~K}$ (green line), $T=500 \mathrm{~K}$ (orange line) and $T=600 \mathrm{~K}$ (purple line) are shown, with respect to the $300 \mathrm{~K}$ values (blue line). Large wavevector phonons (higher q-phonons) have a greater contribution to thermal transport at higher temperatures compared to room temperature (up to $20 \%$ for $T=600 \mathrm{~K}$ ) (Color figure online).

the analysis in Fig. $2 \mathrm{~b}$ also captures the increase in Umklapp scattering, which are competing processes in increasing/reducing the participation of high $\boldsymbol{q}$ states. This makes boundary scattering more dominant in reducing the thermal conductivity at higher temperatures, as we show below in the Monte Carlo simulations.

\section{RESULTS}

We begin our investigation with the effects of nanocrystallinity on the thermal conductivity of $\mathrm{Si}$ as the temperature is raised. In Fig. 3a, the blue line shows the thermal conductivity $\kappa$ for the bulk pristine system (without nanocrystallinity) as a function of temperature from $300 \mathrm{~K}$ to $900 \mathrm{~K}$, which has the typical reduction trend due to the increase in the phonon-phonon scattering rates. At $900 \mathrm{~K}$, for example, there is a decrease in $\kappa$ by more than $60 \%$.

The introduction of nanograin boundaries into the material (as shown in the inset of Fig. 3a) causes an additional reduction in $\kappa$ as expected. We further show in Fig. 3a the $\kappa$ as temperature increases from
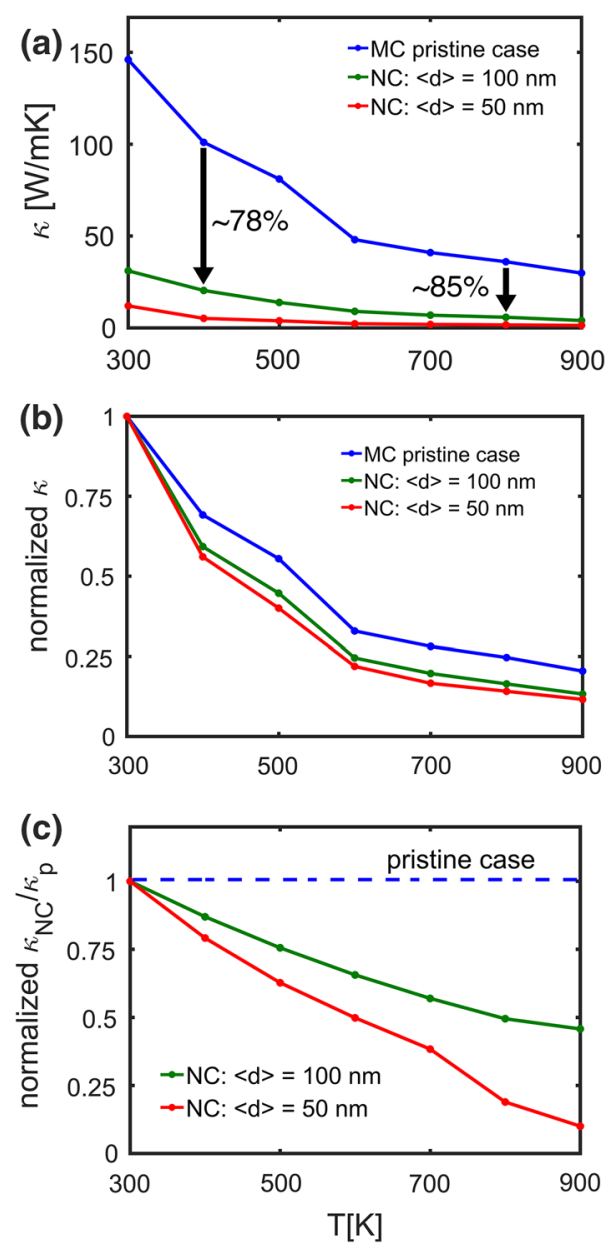

Fig. 3. (a) Effect on thermal conductivity $(\kappa)$ as temperature increases from $300 \mathrm{~K}$ to $900 \mathrm{~K}$, for the pristine case (blue line), nanocrystalline $(N C)$ case with $\langle d\rangle=100 \mathrm{~nm}$ (green line) and $\langle d\rangle=50 \mathrm{~nm}$ (red line); a sharp reduction in $\kappa$ of over $75 \%$ is observed at $300 \mathrm{~K}$ for the $\langle d\rangle=100 \mathrm{~nm}$ case (green line), which drops further to more than $85 \%$ for temperatures over $800 \mathrm{~K}$. The $\kappa$ drop is greater in the $\langle d\rangle=50 \mathrm{~nm}$ case (red line), while a typical geometry for $\langle d\rangle=100 \mathrm{~nm}$ case is given in Fig. 1(a). (b) The data in (a) normalized by the $300 \mathrm{~K} \kappa$ value of the pristine case (blue line); the effect of phonon boundary scattering is removed from all data after this normalization. (c) The data in (a) normalized by the pristine value (blue line) at every temperature; the effect of phonon-phonon scattering from all data is taken away due to this normalization (Color figure online).

$300 \mathrm{~K}$ to $900 \mathrm{~K}$ for nanocrystalline (NC) geometries with $<d>=100 \mathrm{~nm}$ (Fig. 3a, green line) and $<$ $d>=50 \mathrm{~nm}$ (Fig. 3a, red line). A sharp reduction in $\kappa$ of over $75 \%$ is observed at $300 \mathrm{~K}$ for the $<d>=$ $100 \mathrm{~nm}$ case (green line) from the bulk value (blue line) at $300 \mathrm{~K}$. The $\kappa$ drop is greater in the $<d>=$ $50 \mathrm{~nm}$ case (red line) indicating as expected that a smaller grain size causes greater $\kappa$ reduction. Noticeably, however, at elevated temperatures, the reduction in $\kappa$ for the $\langle d\rangle=100 \mathrm{~nm}$ NC structure (Fig. 3a, green line) drops by a larger amount (85\% at $800 \mathrm{~K}$ ). This enhanced reduction of $\kappa$ observed at high temperatures cannot be explained based on phonon-phonon scattering and/or the reduction in $<$ MFP $>$ due to grain boundaries alone, but, as 
we further show below, it is a consequence of the $\boldsymbol{q}$ dependent boundary scattering, which increases as the average phonon $\boldsymbol{q}$-value increases. This overall enhanced $\kappa$ reduction of $\sim 7 \%$ of the pristine value (from $78 \%$ to $85 \%$ due to the $\boldsymbol{q}$-dependent scattering processes) might seem small compared to just the pristine case values; however, in absolute terms, that number is large if compared to the $\kappa$ of the NC case, which stands at just $16 \%$ of the pristine material at $300 \mathrm{~K}$ for $\langle d\rangle=100 \mathrm{~nm}$. Essentially, this $7 \%$ reduction is almost half of the remaining $\kappa$ after the introduction of NC.

To clarify this magnitude, in Fig. 3b, we normalize the thermal conductivity to the $300 \mathrm{~K}$ value for both the single crystal and NC systems (i.e., at $300 \mathrm{~K}$, all geometries begin from the same reference), therefore, essentially removing the effect of the $<$ MFP $>$ reduction due to the grain boundary scattering. The difference in the blue line (pristine) versus the green/red lines (NC structures) is now the difference in the phonon-phonon scattering and the $\boldsymbol{q}$-dependent boundary scattering. By further normalizing $\kappa$ for the $\mathrm{NC}$ geometries by the pristine $\kappa$ value at all temperatures in Fig. 3c, i.e., by computing the $\kappa_{\mathrm{NC}} / \kappa_{\text {pristine }}$ ratio at each temperature (where $\kappa_{\text {pristine }}$ is the blue line in Fig. $3 \mathrm{~b}$ ), we can further eliminate the effect of the temperaturedependent phonon-phonon scattering reduction which is common for all cases. Thus, the differences in the lines of Fig. 3c from the pristine blue dashed line show the influence of the $\boldsymbol{q}$-dependent boundary processes for the NC cases of $\langle d\rangle=100 \mathrm{~nm}$ (green line) and $\langle d>=50 \mathrm{~nm}$ (red line). The $\boldsymbol{q}$ dependent processes are responsible for doubling the reduction in $\kappa$ for the $\langle d\rangle=100 \mathrm{~nm}$ (green line) and amplifying the reduction in $\langle d\rangle=50 \mathrm{~nm}$ (red line) by a factor of four at $900 \mathrm{~K}$. Note that this $\boldsymbol{q}$-dependent scattering at the grain boundaries corresponds to a small decrease in $\kappa$ (compared to the pristine material). In the presence of crystalline boundaries where $\kappa$ is already reduced, this is a large component. As a result, the $Z T$ value of a thermoelectric material will benefit significantly due to this. Note that this effect is built in the boundary scattering, and it is not something that can be easily used directly as a design strategy, but separating its effect from the geometrical features helps the understanding of phonon transport in nanostructures.

Next, we perform the same investigation for hierarchical nanostructured Si-based materials, in which case we incorporate nanopores into the nanocrystalline structures (as in Fig. 1b). A typical simulated geometry for $\phi=5 \%$ porosity with randomly sized and randomly distributed pores is shown in the inset of Fig. 4a. Here, we chose the pore diameter randomly from $D=10 \mathrm{~nm}$ to $D=50 \mathrm{~nm}$ using a uniform distribution. Again, we consider operating temperatures from $T=300 \mathrm{~K}$ to 900 K. In Fig. 4a (blue line), we show the thermal conductivity versus temperature as in Fig. 3a, but
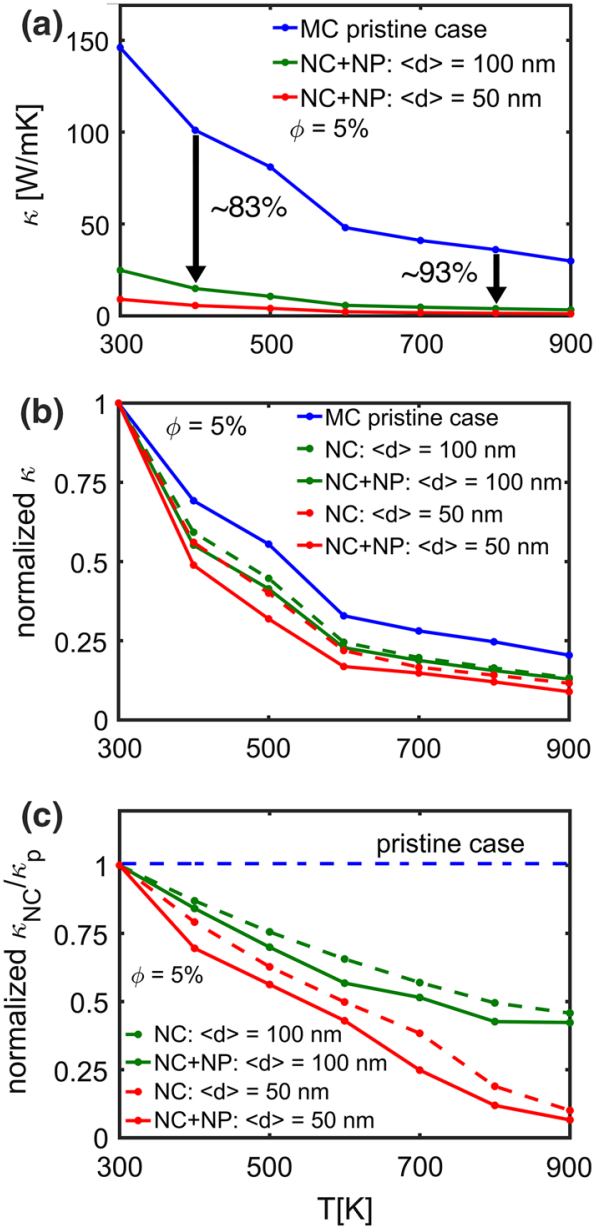

Fig. 4. (a) Effect of hierarchical nanostructures on $\kappa$ as temperature increases from $300 \mathrm{~K}$ to $900 \mathrm{~K}$, for the pristine case (blue line), combined nanocrystalline and nanoporous $(N C+N P)$ case with $\langle d\rangle=100 \mathrm{~nm}$ (green dashed line) with porosity $\phi=5 \%$, and $\langle d\rangle=50 \mathrm{~nm}$ (red dashed line) with porosity $\phi=5 \%$; a reduction in $\kappa$ of over $80 \%$ is observed at $300 \mathrm{~K}$ for the $\langle d\rangle=100 \mathrm{~nm}$ case (green line) and $\kappa$ drops further by more than $90 \%$ for temperatures over $800 \mathrm{~K}$. The $\kappa$ drop is greater in the $\langle d\rangle=50 \mathrm{~nm}$ case (red dashed line). A typical geometry for $\langle d\rangle=100 \mathrm{~nm}$ with $\phi=5 \%$ is shown in in Fig. 1(b). (b) The data in (a) normalized by the $300 \mathrm{~K} \kappa$ value of the pristine case (blue line); the effect of phonon boundary scattering is removed from all data after this normalization. (c) The data in (a) normalized by the pristine value (blue line) at every temperature; the effect of phonon-phonon scattering from all data is taken away due to this normalization. Again, the dashed lines are the data of Fig. 3(c) for the for the NC cases of $\langle d\rangle=100 \mathrm{~nm}$ (green dashed line); $\langle d\rangle=50 \mathrm{~nm}$ (red dashed line) alone. The legend of (b) also applies one-to-one with the lines of (c). At high temperatures, there a further reduction in the normalized ratio observed due to pores (Color figure online).

for the new geometry (the blue line for the pristine material is the same in Fig. 3a). The green and red lines show the behavior of the hierarchical nanostructures (combined nanocrystalline and nanoporous $(\mathrm{NC}+\mathrm{NP})$ case) with $<d>=100 \mathrm{~nm}$ (green dashed line) and $\langle d>=50 \mathrm{~nm}$ (red dashed line). A reduction in $\kappa$ of over $80 \%$ is observed at $300 \mathrm{~K}$ for the $\langle d\rangle=100 \mathrm{~nm}$ case (green line), which is more than the NC only case. $\kappa$ drops further by more than $90 \%$ for temperatures at $800 \mathrm{~K}$. The drop in $\kappa$ is 
even larger in the $\langle d>=50 \mathrm{~nm}$ case (red line) at $\sim 95 \%$.

In Fig. 4b, as earlier in Fig. 3b, we normalize the thermal conductivity of the three structures to their value at $T=300 \mathrm{~K}$. Thus, we remove in this figure the effect of geometry and the MFP of boundary scattering due to the nanostructured features that the phonons encounter. What remains is then the effect of different phonon-phonon MFP (which we do not expect to have much difference) and the $\boldsymbol{q}$ dependence of the boundary scattering. It is clear from this figure that, as the temperature increases, the reduction in the thermal conductivity is larger in the nanocomposite structure, as was also observed earlier. In the dashed lines, we also show the NC lines of Fig. 3b, indicating that the reduction is slightly larger in the hierarchically nanostructured materials.

This is more clearly shown in Fig. 4c, in which we normalize the data lines of Fig. $4 \mathrm{~b}$ to the pristine blue line of Fig. $4 \mathrm{~b}$. This removes the influence of the phonon-phonon scattering, and what is left is just the influence that the $\boldsymbol{q}$-dependence has on the boundary scattering as in Fig. 3c. Again, the $\boldsymbol{q}$ dependence of the boundary scattering has a severe effect, even larger by $\sim 15 \%$ in the case of the hierarchical geometries (solid lines) compared to the nanocrystalline geometries (dashed lines). This is again expected to have a proportional contribution to $Z T$. We note that, while pores themselves do not have any $\boldsymbol{q}$-dependent transmission properties (we treat pore scattering as $\boldsymbol{q}$-independent), their presence introduces more disorder, increases the number of scattering events in the system and the number of times phonons pass through the grain boundaries, thus enhancing the overall effect of $T$ dependent surface scattering. Effectively, pores force phonons to pass from grain boundaries back and forth more times, which increases the importance of the $\boldsymbol{q}$-dependent processes across the boundaries.

In order to directly compare and contrast $\boldsymbol{q}$ dependent and $\boldsymbol{q}$-independent scattering at grain boundaries, we turn off the $\boldsymbol{q}$-dependence and rerun the simulations. In this case, we consider only specular scattering at grain boundary and transmission without change in phonon angle. In Figs. 5a-c, the effect of $\boldsymbol{q}$-independent grain boundary scattering, for the nanocrystalline (NC) case with $<$ $d>=50 \mathrm{~nm}$ (purple dashed line) is compared to the $\boldsymbol{q}$-dependent case (red line). Figure 5a corresponds to same calculations performed in Figs. $3 \mathrm{~b}$ and $4 \mathrm{~b}$, now including the $\boldsymbol{q}$-independent $\langle d\rangle=50 \mathrm{~nm}$ (purple line) calculation. Clearly, the $\boldsymbol{q}$-independent simulation result resides between the pristine and $\boldsymbol{q}$-dependent results through the entire temperature range. Figure $5 \mathrm{~b}$ is equivalent to Figs. $3 \mathrm{c}$ and $4 \mathrm{c}$ and shows the result of removing phonon-phonon scattering effects, indicating the difference between $\boldsymbol{q}$ -
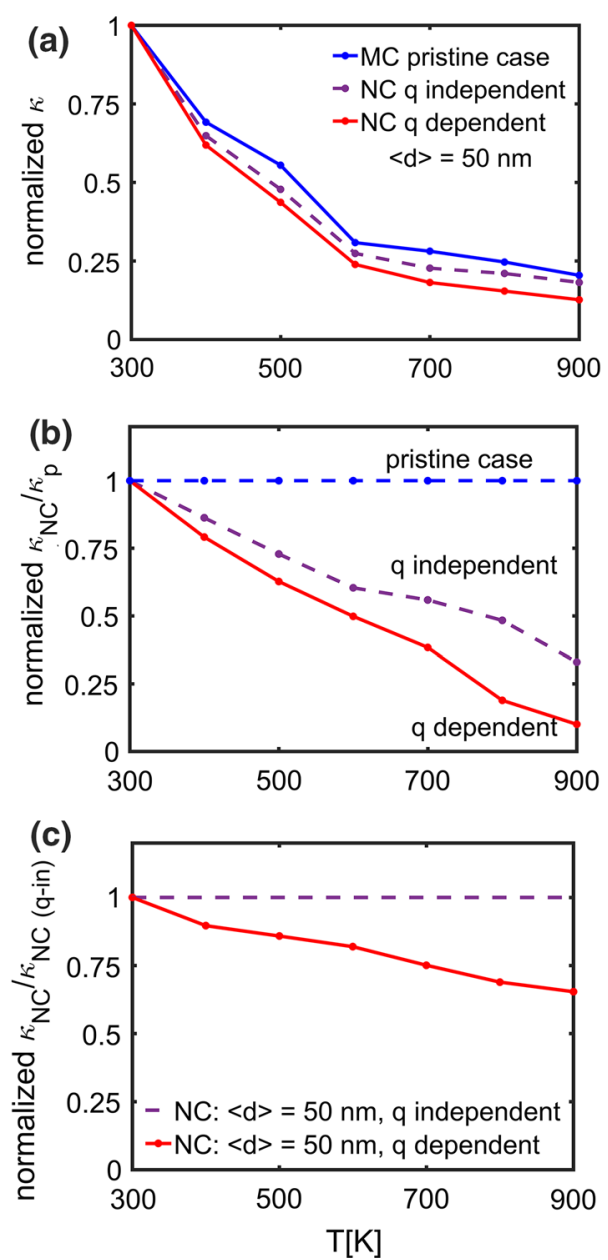

Fig. 5. (a) The effect of $\boldsymbol{q}$-independent and $\boldsymbol{q}$-dependent scattering at grain boundaries compared to the pristine case; all values are normalized with respect to their value at $300 \mathrm{~K}$, and the effect of phonon boundary scattering is removed from all data after this normalization. (b) The data in (a) normalized by the pristine value (blue line) at every temperature; the effect of phonon-phonon scattering from all data is taken away due to this normalization. (c) The data in (b) normalized to the $\boldsymbol{q}$-independent $\mathrm{NC}$ case at all temperatures, showing the effect of the $\boldsymbol{q}$-dependence of the grain boundary scattering as the temperature increases (Color figure online).

dependent and $\boldsymbol{q}$-independent boundary scattering. This is shown more clearly in Fig. 5c, where we normalize the $\boldsymbol{q}$-dependent system to the $\boldsymbol{q}$-independent case (purple dashed line) at $300 \mathrm{~K}$. In the nanocrystalline (NC) case that is $\boldsymbol{q}$-dependent, there is a steady decrease in $\kappa$ as temperature increases, approaching $\mathrm{a} \sim 40 \%$ further reduction in comparison with the $\boldsymbol{q}$-independent case.

\section{CONCLUSIONS}

In this work, we employed the Monte Carlo transport simulator to solve the BTE for phonons in nanocrystalline Si-based materials and nanocrystalline materials with pores. We investigated the influence of wavevector $\boldsymbol{q}$-dependence scattering on 
the nanocrystalline boundaries, and how this affects the thermal conductivity. We show that, at higher temperatures, because the average phonon wavevector $(\boldsymbol{q})$ increases, scattering throughout boundaries becomes stronger, and the thermal conductivity reduction is enhanced with temperature, compared to $\boldsymbol{q}$-independent boundary scattering. We show that, even up to $\sim 40 \%$, further reduction in thermal conductivity at high temperatures $(800 \mathrm{~K})$ is attributed to the $\boldsymbol{q}$-dependence of boundary scattering, compared to if the boundary scattering was $\boldsymbol{q}$-independent. The introduction of random nanopores in addition to nanocrystallinity magnifies this effect by an additional $\sim 15 \%$ at $800 \mathrm{~K}$. This suggests that nanostructuring at high temperatures can actually be more effective than previously thought, and a simple constant MFP due to boundary scattering overestimates the thermal conductivity. We expect that our approach could be used to predict the behavior of materials depicting $\boldsymbol{q}$-dependent boundary scattering at high temperatures, as well as providing insight into the design of low thermal conductivity, high $Z T$ nanostructured materials.

\section{ACKNOWLEDGMENTS}

This work has received funding from the European Research Council (ERC) under the European Union's Horizon 2020 Research and Innovation Programme (Grant Agreement No. 678763). The authors would also like to thank Dr. Patrizio Graziosi at the University of Warwick for useful discussions.

\section{OPEN ACCESS}

This article is distributed under the terms of the Creative Commons Attribution 4.0 International License (http://creativecommons.org/licenses/by/4.0/), which permits unrestricted use, distribution, and reproduction in any medium, provided you give appropriate credit to the original author(s) and the source, provide a link to the Creative Commons license, and indicate if changes were made.

\section{APPENDIX}

\section{Comparison of Single-Phonon Monte Carlo and Literature Data}

The figure below (Fig. 6) compares the singlephonon Monte Carlo thermal conductivity simulation results (blue line) for Si versus temperature to other (multi-phonon) Monte Carlo methods and experimental measured data. Excellent agreement is found.

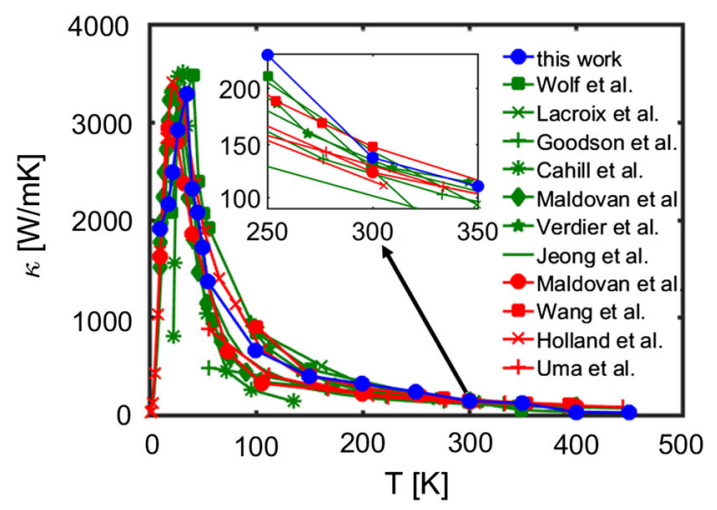

Fig. 6. Validation of the single-phonon Monte Carlo simulator (blue line) for thermal conductivity $(\kappa)$ versus temperature for bulk Si. Simulation works (green lines) ${ }^{15,19,27,31-35}$ and experimental works (red lines) $^{4,26,34,36}$ are shown. The inset shows a close-up of the temperature range between $250 \mathrm{~K}$ and $350 \mathrm{~K}$. The simulated results are in close agreement to other literature results (Color figure online).

\section{REFERENCES}

1. K. Biswas, J. He, Q. Zhang, G. Wang, C. Uher, V.P. Dravid, and M.G. Kanatzidis, Nat. Chem. 3, 160 (2011).

2. K. Biswas, J. He, I.D. Blum, C.-I. Wu, T.P. Hogan, D.N. Seidman, V.P. Dravid, and M.G. Kanatzidis, Nature 489, 414 (2012).

3. B.T. Kearney, B. Jugdersuren, D.R. Queen, T.H. Metcalf, J.C. Culbertson, P.A. Desario, R.M. Stroud, W. Nemeth, Q. Wang, and X. Liu, J. Phys.: Condens. Matter 30, 085301 (2018).

4. Z. Wang, J.E. Alaniz, W. Jang, J.E. Garay, and C. Dames, Nano Lett. 11, 2206 (2011).

5. M.T. Dunham, B. Lorenzi, S.C. Andrews, A. Sood, M. Asheghi, D. Narducci, and K.E. Goodson, Appl. Phys. Lett. 109, 253104 (2016).

6. S. Basu and M. Francoeur, Appl. Phys. Lett. 98, 113106 (2011).

7. J.A.P. Taborda, M.M. Rojo, J. Maiz, N. Neophytou, and M.M. González, Nat. Sci. Rep. 6, 32778 (2016).

8. G. Tan, F. Shi, S. Hao, L.D. Zhao, H. Chi, X. Zhang, C. Uher, C. Wolverton, V.P. Dravid, and M.G. Kanatzidis, Nat. Commun. 7, 12167 (2016).

9. N. Neophytou, X. Zianni, H. Kosina, S. Frabboni, B. Lorenzi, and D. Narducci, Nanotechnology 24, 205402 (2013).

10. N. Neophytou, X. Zianni, H. Kosina, S. Frabboni, B. Lorenzi, and D. Narducci, J. Electron. Mater. 43, 1896 (2014).

11. B. Lorenzi, D. Narducci, R. Tonini, S. Frabboni, G.C. Gazzadi, G. Ottaviani, N. Neophytou, and X. Zianni, J. Electron. Mater. 43, 3812 (2014).

12. S. Foster, M. Thesberg, and N. Neophytou, Phys. Rev. B 96, 195425 (2017).

13. V. Vargiamidis, S. Foster, and N. Neophytou, Phys. Status Solidi A 215, 1700997 (2018).

14. E. Pop, S. Sinha, and K.E. Goodson, J. Electron. Packag. 128, 102 (2006).

15. D. Lacroix, K. Joulain, and D. Lemonnier, Phys. Rev. B 72, 064305 (2005).

16. K. Kukita and Y. Kamakura, J. Appl. Phys. 114, 154312(2013).

17. E. Pop, R.W. Dutton, and K.E. Goodson, J. Appl. Phys. 96, 4998 (2004).

18. S. Mazumdar and A. Majumdar, J. Heat Transfer 123, 749 (2001).

19. S. Wolf, N. Neophytou, and H. Kosina, J. Appl. Phys. 115, 1 (2014). 
20. S. Wolf, N. Neophytou, Z. Stanojevic, and H. Kosina, J. Electron. Mater. 43, 3870 (2014).

21. L.N. Maurer, Z. Aksamija, E.B. Ramayya, A.H. Davoody, and I. Knezevic, Appl. Phys. Lett. 106, 133108 (2015).

22. D. Chakraborty, S. Foster, and N. Neophytou, Phys. Rev. B 98, 115435 (2018).

23. Q. Hao, G. Chen, and M.S. Jeng, J. Appl. Phys. 106, 114321 (2009).

24. L. Weber and E. Gmelin, Appl. Phys. A 53, 136 (1991).

25. S. Ju and X. Liang, J. Appl. Phys. 112, 064305 (2012).

26. M.G. Holland, Phys. Rev. 132, 2461 (1963).

27. C. Jeong, S. Datta, and M. Lundstrom, J. Appl. Phys. 111, 093708 (2012).

28. R. Dettori, C. Melis, X. Cartoixà, R. Rurali, and L. Colombo, Phys. Rev. B 91, 054305 (2015).

29. Z. Aksamija and I. Knezevic, Phys. Rev. B 90, 035419 (2014).

30. H. Karamitaheri, N. Neophytou, and H. Kosina, J. Appl. Phys. 115, 024302 (2014).
31. D.G. Cahill, W.K. Ford, K.E. Goodson, G.D. Mahan, A. Majumdar, H.J. Maris, R. Merlin, and S.R. Phillpot, J. Appl. Phys. 93, 793 (2003).

32. K. Goodson, D.G. Cahill, and A. Majumdar, J. Heat Trans. 124, 223 (2002).

33. M. Verdier, K. Termentzidis, and D. Lacroix, J. Phys.: IOP Conf. Ser. 785, 012009 (2017).

34. M. Maldovan, J. Appl. Phys. 110, 114310 (2011).

35. M. Maldovan, Nature 503, 209 (2013).

36. S. Uma, A.D. McConnell, M. Asheghi, K. Kurabayashi, and K.E. Goodson, Int. J. Thermophys. 22,605 (2001).

Publisher's Note Springer Nature remains neutral with regard to jurisdictional claims in published maps and institutional affiliations. 